\title{
CARACTERIZACION DE ARCILLAS DE LA PROVINCIA DE MORROPON POR TECNICAS FISICAS Y QUIMICAS
}

\author{
Rubén Quille R. ${ }^{1}$, Angel Bustamante D. ${ }^{1,}$, Alejandro Trujillo Q. ${ }^{1}$, Ana María Osorio ${ }^{2}$, \\ Eladio Casapia A. ${ }^{3}$, Carlos Quiñones M. ${ }^{3}$, Clelia Vidal D. ${ }^{3}$ \\ ${ }^{1}$ Facultad de Ciencias Físicas, Universidad Nacional Mayor de San Marcos \\ Apartado Postal 14-0149, Lima, Perú. \\ ${ }^{2}$ Facultad de Química e Ingeniería Química, Universidad Nacional Mayor de San Marcos \\ Apartado Postal 14-0149, Lima, Perú. \\ ${ }^{3}$ Facultad de Ciencias Naturales y Matemática, Universidad Nacional del Callao \\ Apartado Postal 138, Callao, Perú
}

\begin{abstract}
Resumen
La industria cerámica es una actividad muy importante en el distrito de Chulucanas, capital de la provincia de Morropón, y en el distrito de La Encantada. Ambos distritos pertenecen al departamento de Piura, donde se mantiene una gran demanda de arcillas como materia prima para la elaboración de pastas cerámicas. Tradicionalmente han existido evidencias de yacimientos importantes de arcillas; pero, aún no han sido completamente caracterizadas. Así, considerando la importancia de esta materia prima para la industria cerámica de la zona y las dificultades para disponer de materiales cerámicos comerciales de calidad a bajo costo, presentamos en este trabajo los resultados de la caracterización por técnicas físicas y químicas de las arcillas extraídas de la zona.
\end{abstract}

Palabras Claves: Caracterización, yacimientos, arcillas, técnicas físicas y químicas.

\begin{abstract}
The ceramic industry is the most important activity of Chulucanas, capital of the Province of Morropón, and La Encantada District. Both districts belong to the department of Piura, where there is a great demand of clay as raw material for the ceramic paste elaboration. Traditionally have existed evidences of important clay deposits but they have not yet completely characterized. That is way, considering the importance of this raw material for the ceramic industry of the region and the difficulties to get cheap commercial ceramic material of quality; we show the results of the characterization by physics and chemistry techniques of clays extracted from the zone.
\end{abstract}

Keywords: Characterization, deposits, clays, physical and chemistries techniques.

\section{Introducción}

En el Perú, la cerámica de Chulucanas se ha convertido en los últimos años en uno de los principales productos de exportación en el sector de la artesanía, ha sido y es el producto estrella de muchas empresas por sus características en cuanto al acabado, forma, diseño, color y brillo. Chulucanas (figura 1) es un pequeño pueblo situado a una hora de viaje en auto desde la ciudad de Piura, a $1000 \mathrm{~km}$ de la ciudad de Lima, en la costa norte del Perú. Los habitantes de este pueblo son descendientes de los Tallanes, antiguos habitantes precolombinos de esta región, quienes desarrollaron la cerámica en negativo que hoy podemos apreciar en algunos museos bajo el nombre de Vicus. Actualmente existe en Chulucanas el Centro de Investigación Tecnológica y Empresarial (CITE), antes denominado Centro de Desarrollo Artesanal (CEDAR), que provee a los alfareros de arcilla 
extrusada lista para ser trabajada ya sea con torno o paleta. En ningún otro centro alfarero del Perú existe esta facilidad y esto hace pensar que este pueblo se encuentra en una gran ventaja en relación con otros alfareros para lanzarse a la producción a gran escala. La arcilla no es un material específicamente definida, ya que se encuentra constituido por varios tipos de minerales en diversas proporciones, lo que hace necesaria su caracterización para establecer la formulación de pastas que cumplan los requisitos físicos, químicos y mineralógicos considerados en los procesos cerámicos.

La arcilla es una materia prima básica para la industria cerámica, junto con el caolín, la sílice, feldespatos $\mathrm{y}$ aditivos alcalinos $\mathrm{y}$ alcalinotérreos modificadores del punto de fusión [1]. En este trabajo se incluye los resultados obtenidos de la caracterización por técnicas físicas y químicas de las arcillas provenientes de las canteras Ex -Hacienda Huapalas (figura 2), y Carretera Encantada Mogote (figura 3) que se encuentran a $2 \mathrm{~km}$ del distrito de la Encantada, donde se encuentran los talleres cerámicos. Asimismo, se presenta el resultado de la caracterización de la pasta que produce el CITE -Chulucanas.

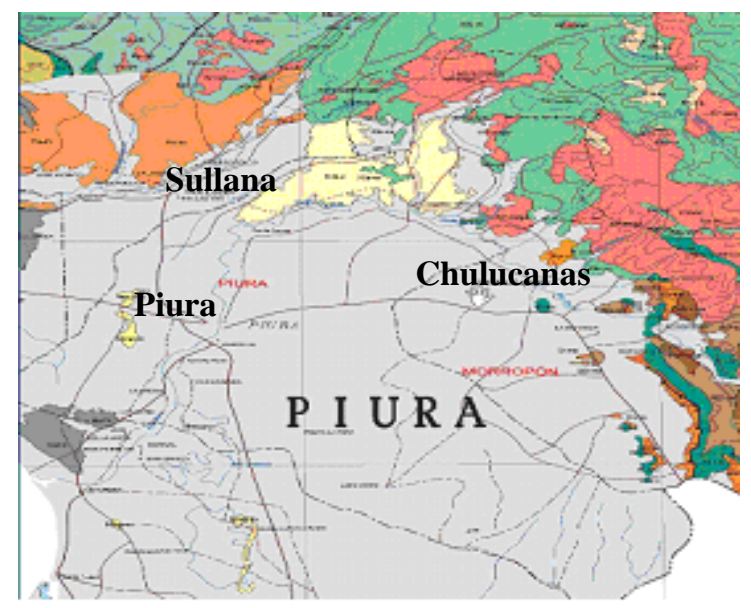

Figura 1. Mapa Geológico de Piura

\section{Parte Experimental}

Para el análisis instrumental de la muestra se procedió de la siguiente manera:

2.1 Preparación de las muestras: Las muestras se sometieron a procesos de cuarteo.
Para los análisis por difracción de rayos $\mathrm{X}$ (DRX), las muestras fueron molidas en un mortero de ágata y pasados por un tamiz de 45 $\mu \mathrm{m}$. Para los análisis por Espectroscopia Mössbauer (EM) se uso $200 \mathrm{mg}$ de material en polvo. Los análisis químicos fueron realizados en el Centro de Microscopia Electrónica de la Universidad Nacional San Agustín de Arequipa usando el Microscopio Electrónico de Barrido (MEB) y EDX. Para la pasta del CITE, se realizó un tratamiento adicional en vista que la muestra se encontraba con un alto porcentaje de humedad y se dejó secar en un horno eléctrico, marca MLW, con resolución de $1{ }^{\circ} \mathrm{C}$, a una temperatura de $50^{\circ} \mathrm{C}$ durante 12 horas, para proseguir con los pasos los pasos antes mencionados.

2.2 Análisis por DRX: Para las mediciones por DRX, se utilizó un difractómetro miniflex "Rigaku", con geometría $(\theta-2 \theta)$. Se uso el método de polvo y los difractogramas se tomaron con radiación $\mathrm{K}_{\alpha}$ del $\mathrm{Cu}$, con una resolución de $0,04 \%$ paso $4 \mathrm{~s}$, y en rango de $5^{\circ}$ $<2 \theta<65^{\circ}$. Para la identificación de los minerales se aplicó el método Hanawalt [2].

2.3 Análisis por MEB: Para la caracterización microestructural se utilizó el Microscopio Electrónico de Barrido Philips SEM XL 20. Para el microanálisis de rayos $\mathrm{X}$ por dispersión de energía se uso el microanalizador de rayos $X$ por energía dispersiva EDAX DX 4i, incorporado al MEB, para identificar la calidad de las muestras. La microscopia electrónica de barrido se basa en el estudio de las señales que emite una muestra a ser bombardeada por un haz de electrones acelerados. La información obtenida de estas señales puede ser muy variada: topografía, estructura y composición de la muestra [3].

2.4 Análisis por EM: Para las mediciones por EM se utilizó un arreglo de transmisión y fueron hechas a temperatura ambiente. Se utilizó una fuente de Co-57. La EM tiene ventajas sobre DRX en todo lo que respecta a la identificación de minerales que contienen hierro por ser EM una técnica isotópicamente selectiva. Los parámetros más relevantes en esos espectros son el desdoblamiento cuadrupolar, el corrimiento isomérico y el 
desdoblamiento magnético hiperfino. Estos son producidos por interacciones hiperfinas del núcleo de hierro con el medio ambiente cristalino pudiéndose identificar los iones $\mathrm{Fe}^{3+}$ y $\mathrm{Fe}^{2+}$ que están presentes llevan los compuestos y para diferentes fases magnéticas [4]. Los espectros Mössbauer fueron ajustados usando el programa NORMOS.

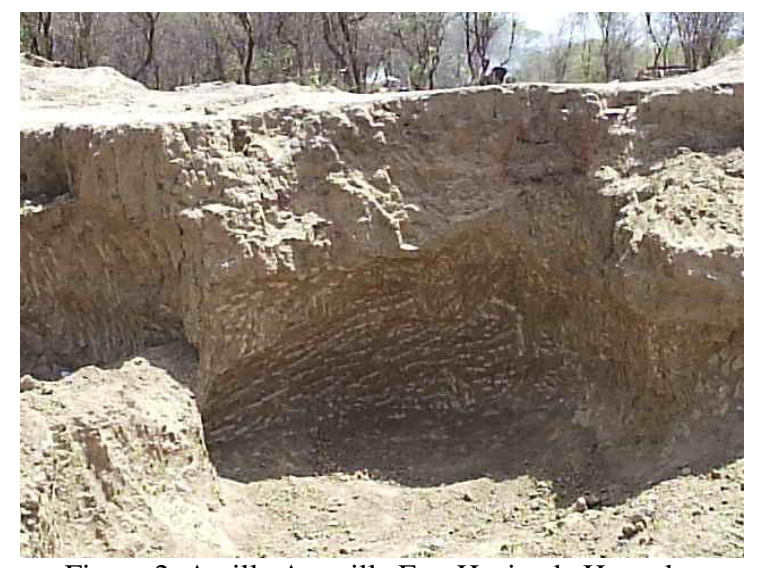

Figura 2. Arcilla Amarilla Ex -Hacienda Huapalas

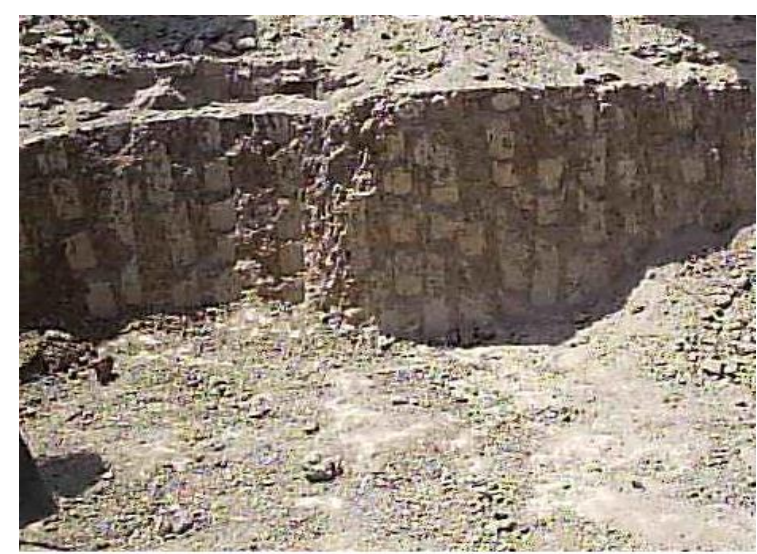

Figura 3. Arcilla Negra Carretera La Encantada -Mogote

\section{Resultados y Discusiones:}

\subsection{Difracción de Rayos $X$}

Los análisis por DRX, de las siguientes muestras de arcillas: Arcilla Amarilla ExHacienda Huapalas (AAEHH), Arcilla Negra Ex-Hacienda Huapalas (ANEHH), Arcilla Amarilla Carretera Encantada-Mogote (AACEM), Arcilla Negra Carretera EncantadaMogote (ANCEM) y la Pasta cerámica elaborada por el CITE (PFCC), a continuación se muestran. Los compuestos minerales identificados se indican en la Tabla 1.

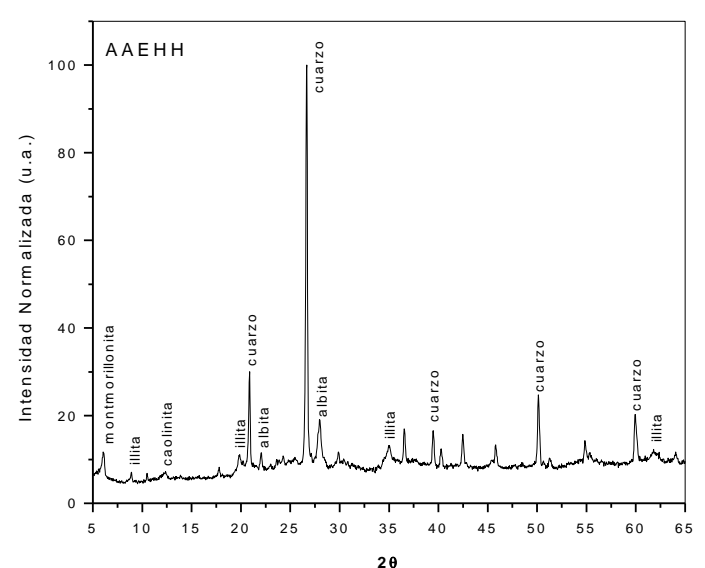

Figura 4. Difractograma correspondiente a AAEHH

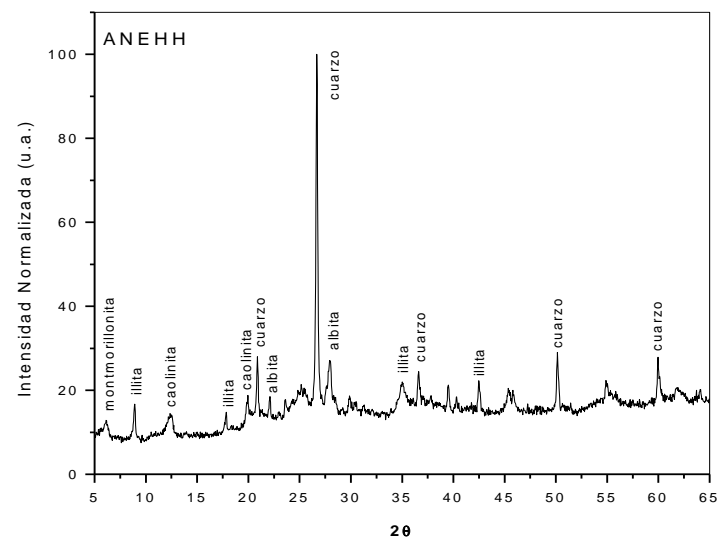

Figura 5. Difractograma correspondiente a ANEHH

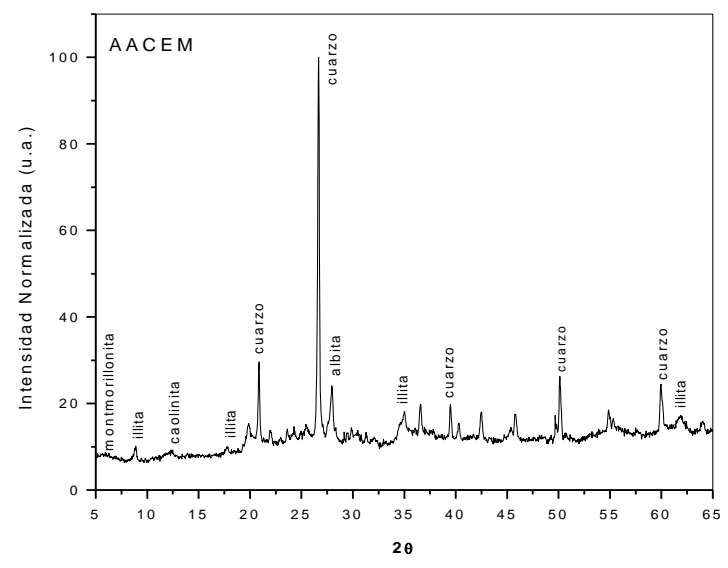

Figura 6. Difractograma correspondiente a AACEM 


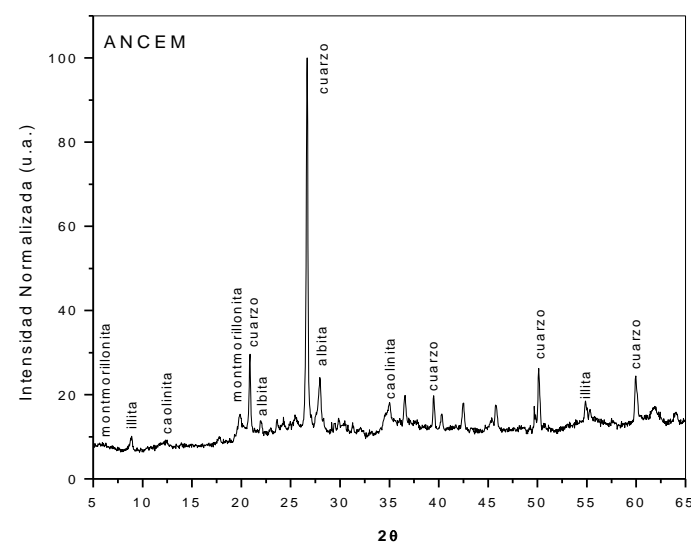

Figura 7. Difractograma correspondiente a ANCEM

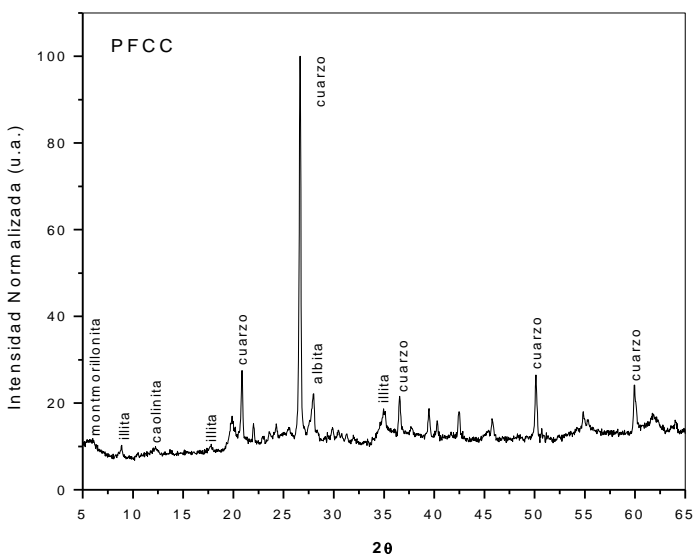

Figura 8. Difractograma correspondiente a la Pasta Cerámica del CITE

Tabla 1. Resultado del análisis mineralógico de las muestras de arcillas

\begin{tabular}{|c|c|c|c|c|c|}
\hline $\mathbf{N}^{\circ}$ & ААЕНН & ANEHH & AACEM & ANCEM & PFCC \\
\hline 1 & $\begin{array}{l}\text { Cuarzo } \\
\mathrm{SiO}_{2}\end{array}$ & $\begin{array}{c}\text { Cuarzo } \\
\mathrm{SiO}_{2}\end{array}$ & $\begin{array}{l}\text { Cuarzo } \\
\mathrm{SiO}_{2}\end{array}$ & $\begin{array}{c}\text { Cuarzo } \\
\mathrm{SiO}_{2}\end{array}$ & $\begin{array}{c}\text { Cuarzo } \\
\mathrm{SiO}_{2}\end{array}$ \\
\hline 2 & $\begin{array}{c}\text { Albita } \\
(\mathrm{Na}, \mathrm{Ca}) \mathrm{Al}(\mathrm{Si}, \mathrm{Al})_{3} \mathrm{O}_{8}\end{array}$ & $\begin{array}{c}\text { Albita } \\
(\mathrm{Na}, \mathrm{Ca}) \mathrm{Al}(\mathrm{Si}, \mathrm{Al})_{3} \mathrm{O}_{8}\end{array}$ & $\begin{array}{c}\text { Albita } \\
\mathrm{NaAl} \mathrm{Si}_{3} \mathrm{O}_{8}\end{array}$ & $\begin{array}{c}\text { Albita } \\
\mathrm{NaAlSi}_{3} \mathrm{O}_{8}\end{array}$ & $\begin{array}{c}\text { Albita } \\
\mathrm{Na}, \mathrm{Ca}) \mathrm{Al}(\mathrm{Si}, \mathrm{Al})_{3} \mathrm{O}_{8}\end{array}$ \\
\hline 3 & $\begin{array}{c}\text { Illita } \\
\mathrm{KAl}_{2} \mathrm{Si}_{3} \mathrm{AlO}_{10}(\mathrm{OH})_{2}\end{array}$ & $\begin{array}{c}\text { Illita } \\
\mathrm{KAl}_{2} \mathrm{Si}_{3} \mathrm{AlO}_{10}(\mathrm{OH})_{2}\end{array}$ & $\begin{array}{c}\text { Illita } \\
\mathrm{KAl}_{2} \mathrm{Si}_{3} \mathrm{AlO}_{10}(\mathrm{OH})_{2} \\
\end{array}$ & $\begin{array}{c}\text { Illita } \\
\mathrm{H}_{2} \mathrm{KAl}_{3} \mathrm{Si}_{3} \mathrm{O}_{12}\end{array}$ & $\begin{array}{c}\text { Illita } \\
\mathrm{KAl}_{2} \mathrm{Si}_{3} \mathrm{AlO}_{10}(\mathrm{OH})_{2}\end{array}$ \\
\hline 4 & $\begin{array}{c}\text { Caolinita } \\
\mathrm{Al}_{2} \mathrm{O}_{3} 2 \mathrm{SiO}_{2} 2 \mathrm{H}_{2} \mathrm{O}\end{array}$ & $\begin{array}{c}\text { Caolinita } \\
\mathrm{Al}_{2} \mathrm{O}_{3} 2 \mathrm{SiO}_{2} 2 \mathrm{H}_{2} \mathrm{O}\end{array}$ & $\begin{array}{c}\text { Caolinita } \\
\mathrm{Al}_{2} \mathrm{O}_{3} 2 \mathrm{SiO}_{2} 2 \mathrm{H}_{2} \mathrm{O}\end{array}$ & $\begin{array}{c}\text { Caolinita } \\
\mathrm{Al}_{2} \mathrm{O}_{3} 2 \mathrm{SiO}_{2} 2 \mathrm{H}_{2} \mathrm{O}\end{array}$ & $\begin{array}{c}\text { Caolinita } \\
\mathrm{Al}_{2} \mathrm{Si}_{2} \mathrm{O}_{5}(\mathrm{OH})_{4}\end{array}$ \\
\hline 5 & $\begin{array}{l}\text { Montmorillonita } \\
\mathrm{NaCaAl} \mathrm{Si}_{4} \mathrm{O}_{12}\end{array}$ & $\begin{array}{l}\text { Montmorillonita } \\
\mathrm{NaCaAl} \mathrm{Si}_{4} \mathrm{O}_{12}\end{array}$ & $\begin{array}{c}\text { Montmorillonita } \\
\mathrm{MgO} \mathrm{Al}_{2} \mathrm{O}_{3} 5 \mathrm{SiO}_{2} \\
\mathrm{H}_{2} \mathrm{O}\end{array}$ & $\begin{array}{c}\text { Montmorillonita } \\
\mathrm{MgO} \mathrm{Al}_{2} \mathrm{O}_{3} 5 \mathrm{SiO}_{2} \\
\mathrm{H}_{2} \mathrm{O}\end{array}$ & $\begin{array}{c}\text { Montmorillonita } \\
\mathrm{Mg} \mathrm{OAl}_{2} \mathrm{O}_{3} 5 \mathrm{SiO}_{2} \\
\mathrm{H}_{2} \mathrm{O}\end{array}$ \\
\hline
\end{tabular}

Tabla 2. Resultados del análisis químico de la muestras de arcillas

\begin{tabular}{|c|c|c|c|c|c|}
\hline Composición & $\begin{array}{c}\text { AAEHH } \\
(\boldsymbol{\%})\end{array}$ & $\begin{array}{c}\text { AACEM } \\
(\boldsymbol{\%})\end{array}$ & $\begin{array}{c}\text { ANEHH } \\
(\boldsymbol{\%})\end{array}$ & $\begin{array}{c}\text { ANCEH } \\
(\boldsymbol{\%})\end{array}$ & $\begin{array}{c}\text { PFCC } \\
(\boldsymbol{\%})\end{array}$ \\
\hline $\mathrm{Na}_{2} \mathrm{O}$ & 2,01 & 1,36 & 0,84 & ----- & 1,07 \\
\hline $\mathrm{MgO}$ & 4,05 & 2,82 & 2,38 & 2,27 & 3,00 \\
\hline $\mathrm{Al}_{2} \mathrm{O}_{3}$ & 21,33 & 22,53 & 26,14 & 20,79 & 24,26 \\
\hline $\mathrm{SiO}_{2}$ & 61,53 & 61,44 & 50,80 & 54,43 & 58,91 \\
\hline $\mathrm{K}_{2} \mathrm{O}$ & 1,71 & 3,04 & 2,77 & 2,97 & 2,16 \\
\hline $\mathrm{CaO}$ & 1,70 & 0,89 & 1,45 & 3,63 & 1,70 \\
\hline $\mathrm{TiO}_{2}$ & 0,64 & 0,69 & 1,07 & 1,54 & 0,61 \\
\hline $\mathrm{Fe}_{2} \mathrm{O}_{3}$ & 7,03 & 7,23 & 12,80 & 13,91 & 8,29 \\
\hline $\mathrm{Cl}_{2} \mathrm{O}$ & ----- & ----- & 0,08 & 0,25 & ----- \\
\hline $\mathrm{MnO}$ & ---- & ----- & 1,67 & 0,21 & ----- \\
\hline Total & $\mathbf{1 0 0}$ & $\mathbf{1 0 0}$ & $\mathbf{1 0 0}$ & $\mathbf{1 0 0}$ & $\mathbf{1 0 0}$ \\
\hline
\end{tabular}

Según los difractogramas de la figura 4 a la figura 8 , vemos que la fase mineralógica caolinita está presente en las cuatro muestras de arcillas, asimismo las fases de cuarzo, albita, montmorillonita e illita. 


\subsection{Microscopia Electrónica de Barrido}

Según los resultados de la microscopia electrónica de barrido de la Tabla 2. Los materiales arcillosos presentan baja concentración de óxidos de hierro entre $7 \%$ y $13 \%$, esto es una ventaja para la preparación de cuerpos blancos. Asimismo, podemos notar la buena presencia de alumina entre $20 \%$ a 26 $\%$, y silicatos entre $50 \%$ y $61 \%$. Esto favorece en la preparación del cerámico ya que las arcillas tienen baja plasticidad.

\subsection{Espectroscopia Mossbauer}

\subsubsection{Muestra: Arcilla amarilla ex - hacienda Huápalas, AAEHH}

La Figura 9 muestra el espectro Mössbauer (EM) a temperatura ambiente y en régimen de alta velocidad de la muestra denominada AAEHH de la cantera ex hacienda Huápalas, en la que se observa la presencia de cuatro dobletes paramagnéticos, correspondiendo dos dobletes a $\mathrm{Fe}^{2+}$ y dos a $\mathrm{Fe}^{3+}$. No se registro ninguna componente magnética, procediéndose a tomarse un espectro a baja velocidad como se muestra en la Figura 10. Los parámetros hiperfinos asociados con esta muestra se dan en la Tabla 3.

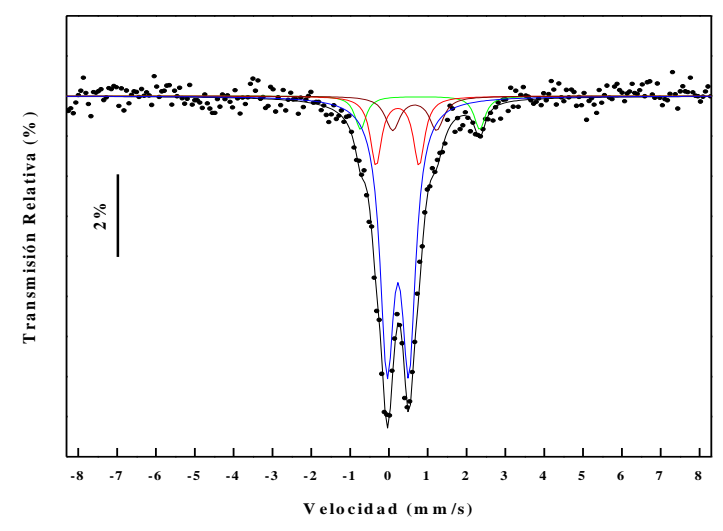

Figura 9. EM a temperatura ambiente $y$ en alta velocidad de AAEHH

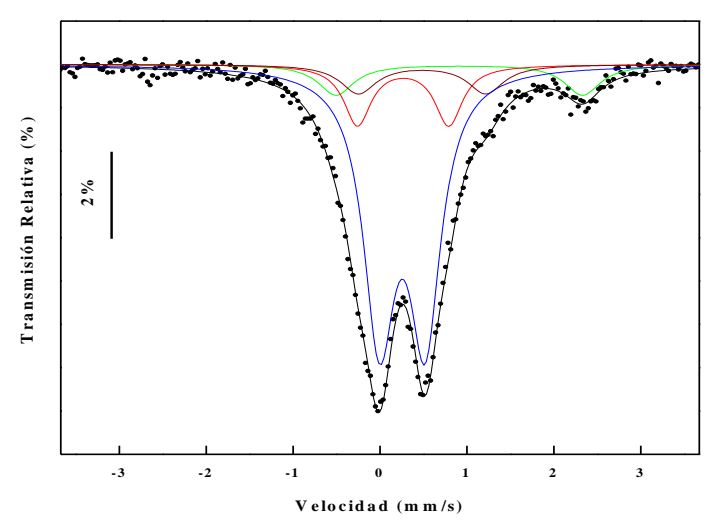

Figura 10. EM a temperatura ambiente y en baja velocidad de AAEHH

\subsubsection{Muestra: Arcilla negra ex - hacienda Huápalas, ANEHH}

La Figura 11 muestra el espectro Mössbauer a temperatura ambiente y en régimen de alta velocidad de la muestra denominada ANEHH de la cantera Ex Hacienda Huápalas, en la que se observa la presencia de cuatro dobletes paramagnéticos, correspondiendo dos dobletes a $\mathrm{Fe}^{2+}$ y dos a $\mathrm{Fe}^{3+}$. También se observa una componente magnética debida a la presencia de un óxido o hidróxido. A fin de resolver mejor la parte central del espectro se procedió a tomarse un espectro a baja velocidad como se muestra en la Figura 12.

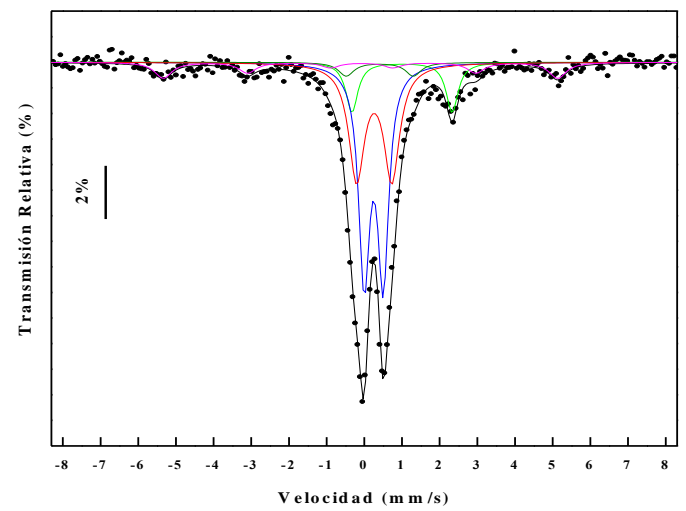

Figura 11. EM a temperatura ambiente $y$ en alta velocidad de ANEHH

Observamos mejor en el espectro de la Figura 12, que la línea ensanchada del doblete rojo corresponde a la presencia de partículas de óxido tan pequeñas a $100 \AA$, debido a la rápida fluctuación térmica de la magnetización, 


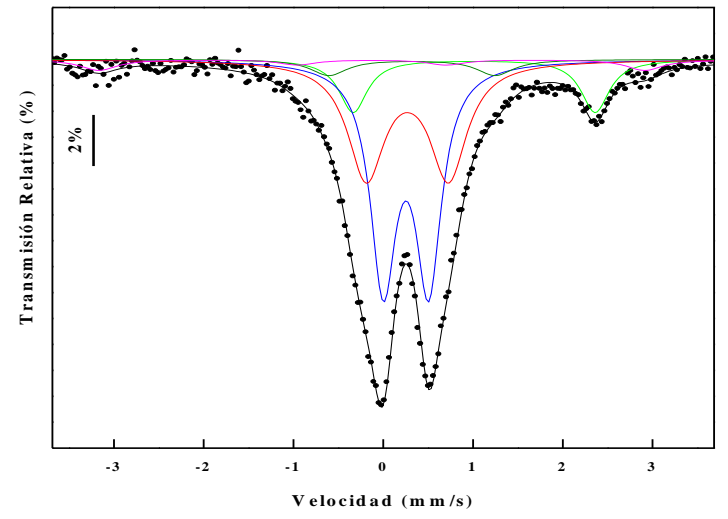

Figura 12. EM a temperatura ambiente y en baja velocidad de ANEHH

un efecto denominado super paramagnetismo y forma parte del sexteto observado a alta velocidad, una medida a helio liquido se hace necesario para una mejor identificación del óxido o hidróxido.

\subsubsection{Muestra: Arcilla amarilla carretera Encantada - Mogote, AACEM}

El espectro Mössbauer a alta velocidad de la muestra AACEM no registra ninguna componente magnética tal como se observa en la Figura 13. El ajuste realizado indica la presencia de cuatro dobletes paramagnéticos, correspondiendo dos dobletes a $\mathrm{Fe}^{2+}$ y dos a $\mathrm{Fe}^{3+}$, procediéndose a tomar el respectivo espectro a baja velocidad como se observa en la Figura 14.

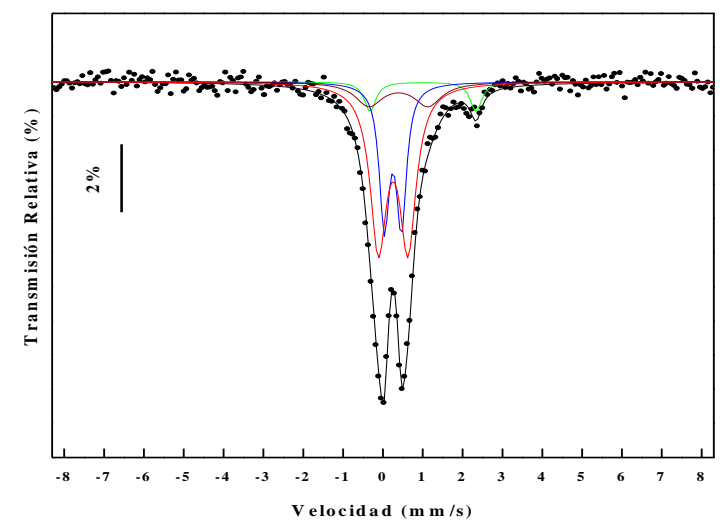

Figura 13. EM a temperatura ambiente y en alta velocidad de AACEM

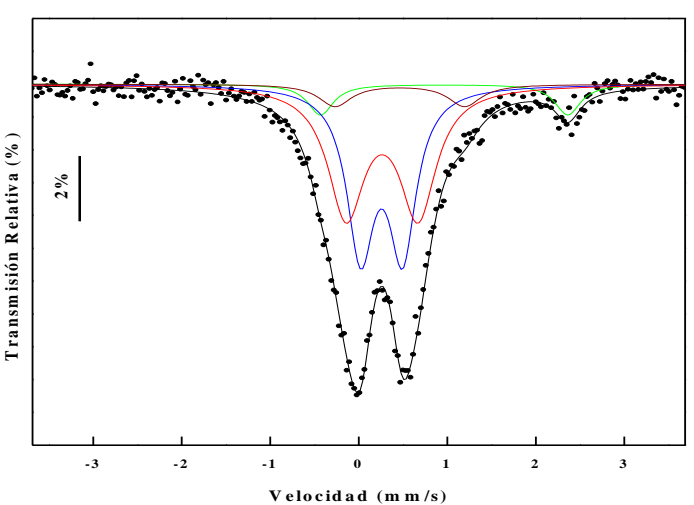

Figura 14. EM a temperatura ambiente y en baja velocidad de AACEM

\subsubsection{Muestra: Arcilla negra carretera Encantada - Mogote, ANCEM}

El espectro Mössbauer a alta velocidad de la muestra ANCEM registra una pequeña componente magnética que representa el $9 \%$ del área total (Tabla 3) como se observa en la Figura 15 y tres dobletes, procediéndose a tomar el respectivo espectro a baja velocidad como se observa en la Figura 16.

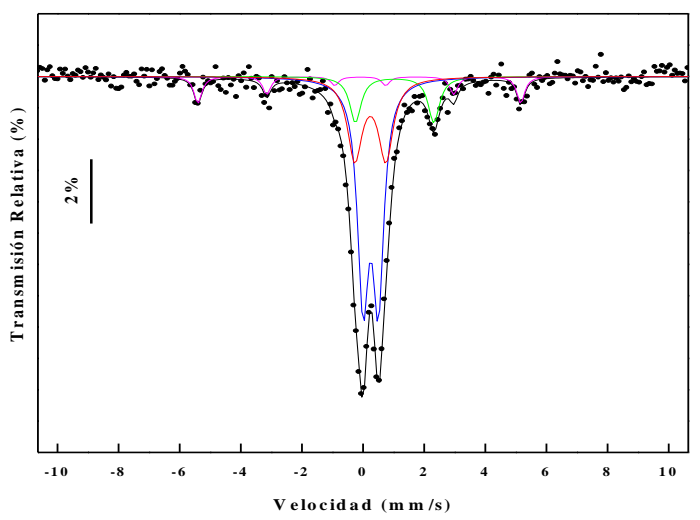

Figura 15. EM a temperatura ambiente y en alta velocidad de ANCEM

\subsubsection{Muestra: Pasta final del CITE - Chulucanas, PFCC}

El material mas importante corresponde a esta muestra PFCC, por ser una pasta formulada y desarrollada en el CITE Chulucanas. El espectro Mössbauer medido en alta velocidad no registra ninguna componente magnética (Figura 17), solo presenta cuatro dobletes, dos paramagnéticos correspondientes a $\mathrm{Fe}^{2+}$ y dos 


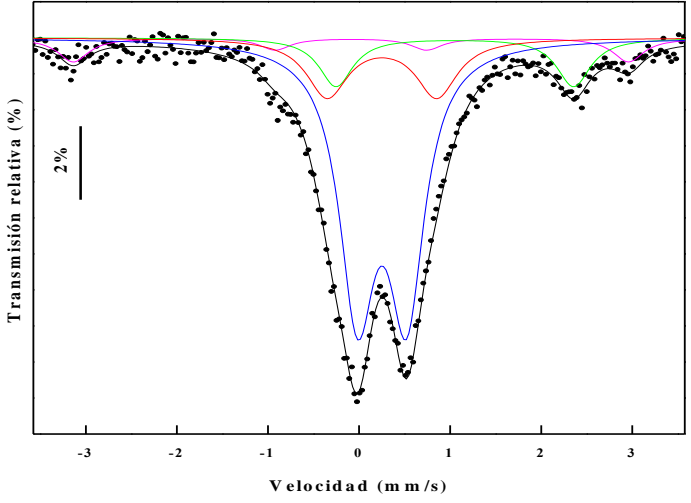

Figura 16. EM a temperatura ambiente y en baja velocidad de ANCEM

a $\mathrm{Fe}^{3+}$. Con la finalidad de resolver mejor el espectro se bajo la velocidad como se observa en la Figura 18, y se observa que el doblete rojo tiene un ancho de línea ensanchada característico de la presencia de partículas pequeñas de óxidos o hidróxidos menores que $100 \AA$ A debido al efecto superparamagnético. Una medida a baja temperatura se hace necesario para un mejor análisis

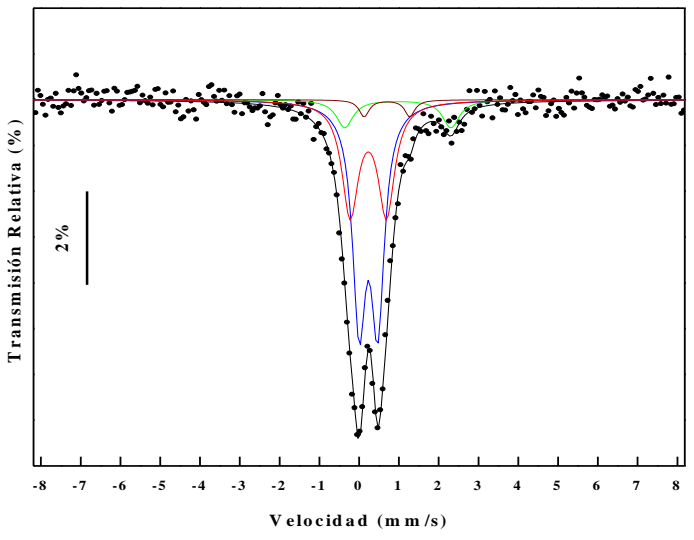

Figura 17. EM a temperatura ambiente y en alta velocidad de PFCC

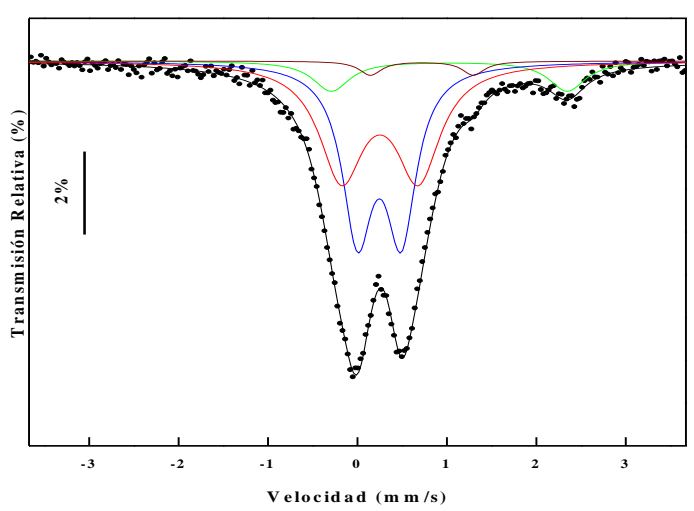

Figura 18. EM a temperatura ambiente y en baja velocidad de PFCC

Tabla 3. Parámetros Mössbauer de las muestra de arcillas, medidos a temperatura ambiente. IS = Corrimiento isomérico en mm/s relativo al Fe metálico, $\mathrm{QS}=$ Desdoblamiento cuadrupolar en $\mathrm{mm} / \mathrm{s}, \mathrm{BH}_{\mathrm{f}}=$ Campo magnético hiperfino en Tesla, $\Gamma=$ ancho de línea en $\mathrm{mm} / \mathrm{s}$ y $\mathrm{A}=$ Área relativa (\%).

\begin{tabular}{|c|c|c|c|c|c|c|c|c|c|c|c|c|c|}
\hline \multirow[t]{2}{*}{ Muestras } & \multicolumn{3}{|c|}{$\mathrm{Fe} 2+(\mathrm{A})$} & \multicolumn{3}{|c|}{$\mathrm{Fe} 2+(\mathrm{D}) /(\mathrm{E})$} & \multicolumn{4}{|c|}{$\mathrm{Fe}^{3+}(\mathrm{B}) /(\mathrm{C})$} & \multicolumn{3}{|c|}{ H1 } \\
\hline & IS & QS & $\Gamma \quad \mathbf{A}$ & IS & QS & $\Gamma \quad \mathbf{A}$ & IS & QS & $\Gamma$ & & $\mathrm{BH}_{\mathrm{f}} \quad \mathrm{IS}$ & QS & $\Gamma \quad \mathbf{A}$ \\
\hline AACEM & 1.120 & 2.66 & 0.3507 & 0.505 & 1.48 & 0.8313 & $\begin{array}{l}0.36 \\
0.370\end{array}$ & $\begin{array}{l}40.43 \\
0.74\end{array}$ & $\begin{array}{l}0.30 \\
0.50\end{array}$ & $\begin{array}{l}28 \\
53\end{array}$ & & & \\
\hline ANCEM & 1.146 & 2.57 & 0.4512 & & & & $\begin{array}{l}0.363 \\
0.35\end{array}$ & $\begin{array}{l}30.50 \\
1.02\end{array}$ & $\begin{array}{l}0.44 \\
0.58\end{array}$ & $\begin{array}{l}52 \\
27\end{array}$ & 32.760 .006 & -0.015 & 0.3109 \\
\hline AАEHH & 0.917 & 3.07 & 0.3507 & 0.776 & 1.13 & 0.4510 & $\begin{array}{l}0.345 \\
0.337\end{array}$ & $\begin{array}{l}50.56 \\
1.11\end{array}$ & $\begin{array}{l}0.43 \\
0.35\end{array}$ & $\begin{array}{l}68 \\
15\end{array}$ & & & \\
\hline ANEHH & 1.115 & 2.66 & $0.38 \quad 11$ & 0.523 & 1.78 & 0.5004 & $\begin{array}{l}0.363 \\
0.377\end{array}$ & $\begin{array}{l}30.51 \\
70.94\end{array}$ & $\begin{array}{l}0.35 \\
0.50\end{array}$ & $\begin{array}{l}42 \\
33\end{array}$ & $\begin{array}{ll}32.46 & 0.028\end{array}$ & -0.016 & 0.5510 \\
\hline PFCC & 1.086 & 2.67 & 0.5109 & 0.812 & 1.15 & 0.3003 & $\begin{array}{l}0.348 \\
0.34\end{array}$ & $\begin{array}{r}0.47 \\
+0.91 \\
\end{array}$ & $\begin{array}{l}0.40 \\
0.50\end{array}$ & $\begin{array}{l}53 \\
35\end{array}$ & & & \\
\hline
\end{tabular}




\section{Conclusiones}

- El análisis de Difracción de Rayos X en las muestras de Chulucanas determinaron la presencia de cuarzo seguido por albita, luego algunos minerales arcillosos. No se notaron grandes diferencias entre ellas.

- La aplicación de la microscopia electrónica de barrido (MEB) nos ayudó a la determinación de compuestos químicos que se encuentra en cada muestra. Según los resultados nos indica que hay mayor presencia de alúmina y oxido de silicio esto nos indica que las muestras de arcilla no tienen demasiada plasticidad.

- Mediante la espectroscopia Mössbauer (EM) se determinaron los parámetros Mössbauer, las muestras presentaron bajo contenido de hierro, aspecto deseable para la elaboración de cuerpos cerámicos blancos.

\section{Agradecimientos}

Las mediciones por EM y DRX se llevaron a cabo en el Laboratorio de Espectroscopia Mössbauer y Difracción de Rayos X de la Facultad de Ciencias Físicas de la Universidad Nacional Mayor de San Marcos, y para el análisis químico las muestras se enviaron al Centro de Microscopia Electrónica de la Facultad de Ingeniería de Procesos de la Universidad Nacional San Agustín de Arequipa. Se agradece la colaboración del grupo de investigadores del Proyecto "Mejora en la calidad de los productos cerámicos para la exportación de los artesanos de Ayacucho, Arequipa y Huancavelica usando técnicas físicas y químicas. I Etapa". Expresamos nuestro agradecimiento al CONCYTEC por el apoyo brindado para el desarrollo de este proyecto

\section{Referencias}

[1] R.W. Grimshaw, "The Chemistry and Physics of Cáliz”, E. Benn Ltd., 4a ed., London (1971).

[2] R.A. Quille, A.G. Bustamante "Análisis Mineralógico Cualitativo y Cuantitativo en Arcillas por difracción de rayos $\mathrm{X}$ y el Método Rietveld usando el programa Fullprof" Revista de Investigación de Física, Vol. $9, \mathrm{~N}^{\circ} 1$ p. 64-68 UNMSM (2006).

[3] P. Escribano, J.B. Carda, E. Cordoncillo. "Esmaltes y Pigmentos Cerámicos", editorial Faenza Editrice Ibérica s.I., España (2001).

[4] M.L. Cerón, J. Fabián, y J. Bravo "Estudio mineralógico de suelos agrícolas por espectroscopia Mössbauer" Revista de Investigación de Física Vol. 2 UNMSM, (1999). 